\title{
Synthesis of high molecular weight polyimide consisting hexafluoroisopropylidene moiety for gas separation
}

\author{
N.A. Mokri, P.C. Oh* ${ }^{*}$ H. Mukhtar and T.L. Chew \\ ${ }^{1}$ Faculty of Chemical Engineering, Universiti Teknologi PETRONAS, \\ 32610 Seri Iskandar, Malaysia \\ *Email: peiching.oh@utp.edu.my \\ Phone: +6053687568; Fax: +6053656176
}

\begin{abstract}
In this work, high molecular weight of heterocyclic aromatic polyimide consisting hexafluoroisopropylidene moiety in both diamine and dianhydride fragments was synthesised. The synthesis was performed via a two-step polycondensation route, which involved polyamic acid formation and chemical imidization. Subsequently, physical purification technique was conducted by microfiltration and re-crystallisation to obtain oligomers-free high molecular weight polymer. Selections of high purity and suitable solvent, type of amine catalyst, precipitation medium and reaction temperature were considered to favour the formation of high molecular weight polyimide. The synthesised polyimide was characterised by ATR-FTIR, which confirmed the presence of significant imide bands denoting the formation of imide linkage. Further confirmation of polyimide molecular structure was accomplished using ${ }^{13} \mathrm{C}$ and ${ }^{1} \mathrm{H}$ NMR. It was found that polyimide with high molecular weight $(4500 \mathrm{KDa})$ was obtained with a polydispersity index of 1.48. Therefore, this polymer has favourable properties for gas separation membrane application.
\end{abstract}

Keywords: Fluorinated polyimide; chemical imidization; hexafluoroisopropylidene; gas separation.

\section{INTRODUCTION}

Nowadays, a wide range of glassy polymeric materials especially polyimides (PIs) are extensively explored in multifarious applications including gas separation for acid gas removal, hydrogen recovery and biogas applications. Fluorinated polyimide (FPI) i.e 6FDA-6FpDA is a type of heterocyclic aromatic polyimides which contains bulky fluorine fragments $\left(-\mathrm{CF}_{3}\right)_{2}$, serving as a non-planar, twisted structure in the main branch of polymer [1-3]. Among the aromatic polyimide class, fluorine-containing polyimide seems very popular among scientists because of its superior characteristics such as thermo-oxidative capability [4], high chemical resistance [5], low refractive index [6, 7], low water absorption [6, 8], soluble in a range of organic solvents [9], high permselectivity [10-12] and fair resistance to plasticization [13, 14]. These aforementioned characteristics explained why this class of polymer is a promising candidate for gas separation application. This synthetic polymer is a more suitable material for chemical transport as compared to the naturally occurring polymer due to its hydrophobicity and inertness to chemical reaction $[6,15,16]$. Additionally, the increase in permselectivity was clearly explained from atomistic studies done by Pandiyan and his 
co-workers $[12,14,17-20]$. It has been shown that the repeat fragment of hexafluoroisopropylidene group $\mathrm{R}-\mathrm{C}\left(\mathrm{CF}_{3}\right)_{2}-\mathrm{R}$, can increase both permeability and selectivity. The properties of bulky fluorine fragments in dianhydride and diamine brought significant changes to permselectivity as the substituent, $\mathrm{R}-\mathrm{C}\left(\mathrm{CF}_{3}\right)_{2}-\mathrm{R}$ existed in the polymer's segmental backbone. The effects on bulk properties can be observed in dianhydride in terms of permeability and solubility while it causes material improvement when incorporated into diamine. In fact, the fluorine linkages $(6 \mathrm{~F})$ are responsible for changes in effective packing as well as accessible free volume (FFV). The change in effective packing is associated with reduction in polymer segmental mobility, interchain interaction and an increase in FFV (0.175-0.190) as reported in literatures [10-12, 17, 19, $21,22]$. Due to the presence of 1,3-trifluoromethyl side groups in diamine and dianhydride segmental chains, it has led to restricted torsional motion of neighbouring phenyl rings and introduction of small free-volume holes. Therefore, 6FDA consisting 2bis (4-aminophenyl) hexafluoropropane diamine (6FpDA) will give some advantages i.e., chemical affinity and polarisability towards polar gases like $\mathrm{CO}_{2}, \mathrm{O}_{2}$ and $\mathrm{N}_{2}$. The affinity of gas molecules will form polar-polar interaction with the delocalised character of the bulky group, thereby increasing the percentage of gas retention. Thus, this will result in excellent gas permeability. Additionally, the presence of this fluorine linkage in the hexafluoroisopropylidene moiety will also increase the processability of polymer due to its solubility in various polar organic solvents.

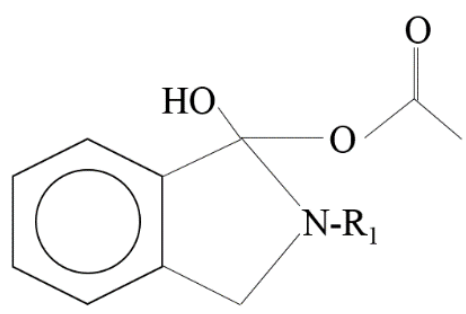

Figure 1. Isoimide (i.e., iminolactone structure).

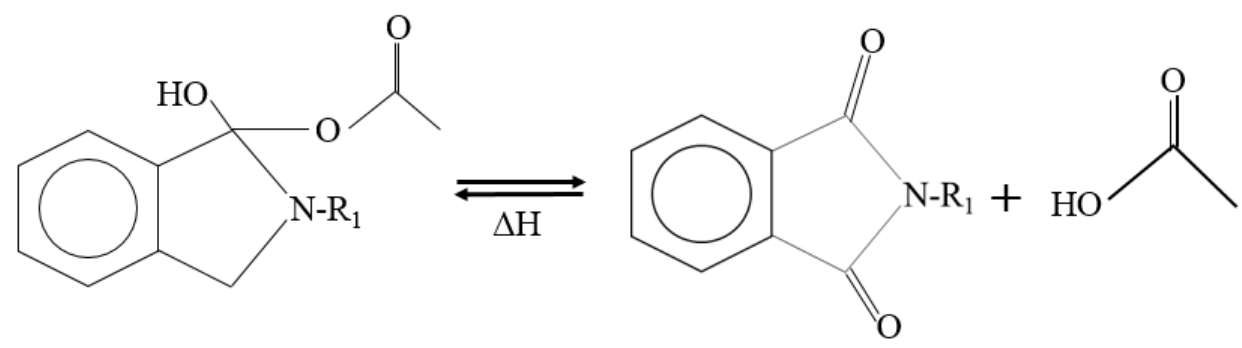

Figure 2. Isoimide conversion to imide after heat treatment.

High molecular weight FPIs basically reflect the degree of imidization (closedring structure of imide repeating unit) after polycondensation reaction is completed. Higher imidization degree of FPIs is needed to form polymeric membrane with the desired permeation properties and mechanical strength. Thus far, polycondensation reaction via catalytic conversion using chemical reagents (chemical imidization) was reported to yield lower molecular weight of PIs as compared to thermal conversion [23, 24]. In the chemical imidization stage where polycondensation of polyamic acid (PAA) to polyimide (PI) occurs, there is a possibility of incomplete cyclisation and also isoimide 
formation (Figure 1). Therefore, thermal treatment at high temperature below the $\mathrm{T}_{\mathrm{g}}$ of FPI will direct the rearrangement of isoimide to imide and also thermally cyclise the amide unit (Figure 2). Hence, high molecular weight of FPIs will be achieved as the degree of imidization also increases. In this work, high $\mathrm{M}_{\mathrm{w}}$ FPIs were synthesised by a classical two-step polycondensation via chemical imidization. Chemical conversion of polyamic acid to polyimide repeating unit is favoured under the right composition of imidising mixture. It is hypothesised that the degree of imidization can further be improved with post-thermal treatment of polymer precipitates. Therefore, the assynthesised FPIs has experienced a sufficient thermal treatment to fully convert isoimides to imides. It is also suggested that further polymer purification is crucial to remove undesired components such as excess reactants as this can affect the polymer $\mathrm{M}_{\mathrm{w}}$ distribution. Therefore, 6FDA-6FpDA-FPIs has been successfully synthesised using the chemical imidization route.

\section{METHODS AND MATERIALS}

\section{Experimental Setup}

N-Methyl-2-pyrrolidone (NMP), calcium hydride, methanol and acetic anhydride were supplied by Merck, 4,4'-(hexafluoroisopropylidene) diphthalic anhydride (6FDA) (assay: 99.9\%) and $\beta$-picoline (assay: 99\%) were purchased from Aldrich, 4,4' (hexafluoroisopropylidene) dianiline (6FpDA) (assay: 98\%) was supplied by Acros Organic and tetrahydrofuran (THF) was purchased from Fisher Scientific. The polymer synthesis was conducted in an inert atmosphere under nitrogen at room temperature. The route of polyimide synthesis is clearly shown in a mechanistic pathway (Figure 3 ). In the two-step polycondensation, formation of polyamic acid was developed by the addition of monomer into anhydrous NMP. The pre-dried diamine monomer (6FpDA) was added into the reaction solvent until complete dissolution. After that, the pre-dried dianhydride monomer (6FDA) was added in 5 aliquots as 6FDA slowly dissolved in NMP due to nucleophilic attack for the ring opening of diamine (Figure 4). This step is the most crucial step for propagation of polyamic acids. About $0.2 \mathrm{~g}$ of dianhydride monomer was added in excess to result in dianhydride end-capping PI. During $24 \mathrm{~h}$ of PAAs formation, extremely dry and high concentration mixture needed to be ensured so that the depropagation step or reversible reaction can be minimised. The formation of polyamic amic (PAA) was conducted for $24(\mathrm{~h})$ as it will give the highest molecular weight $\left(\mathrm{M}_{\mathrm{w}}\right)$ as reported in literature [17]. Cyclisation of PAAs was conducted via chemical imidization using a pyridine-based dehydrating catalyst ( $\beta$-picoline, pKa 5.68 at $20^{\circ} \mathrm{C}$ ) and dehydrating agent i.e., acetic anhydride. This combination will give a complex counter ion which will naturally attack the poor electron deficient group in a mechanistic way (i.e., tautomerization, $\mathrm{A}$ and $\mathrm{B}$ ).

After $12 \mathrm{~h}$ of chemical reaction, the polymer solution was poured into methanol in a beaker and precipitates were recovered from the methanol solution via filtration. The off-white coloured precipitates were obtained and dried overnight in a vacuum oven prior to thermal treatment at an elevated temperature of $150^{\circ} \mathrm{C}$ for $12 \mathrm{~h}$. Purification technique via re-crystallisation was conducted to remove impurities by re-dissolving the solid polymer in tetrahydrofuran and filtering using $1 \mu \mathrm{m}$ nylon membrane filter. A thick, viscous yellow coloured polymer gel was obtained and left for $24 \mathrm{~h}$ at ambient condition to remove the solvent. The polymer was further dried at $60^{\circ} \mathrm{C}$ prior to storage. 

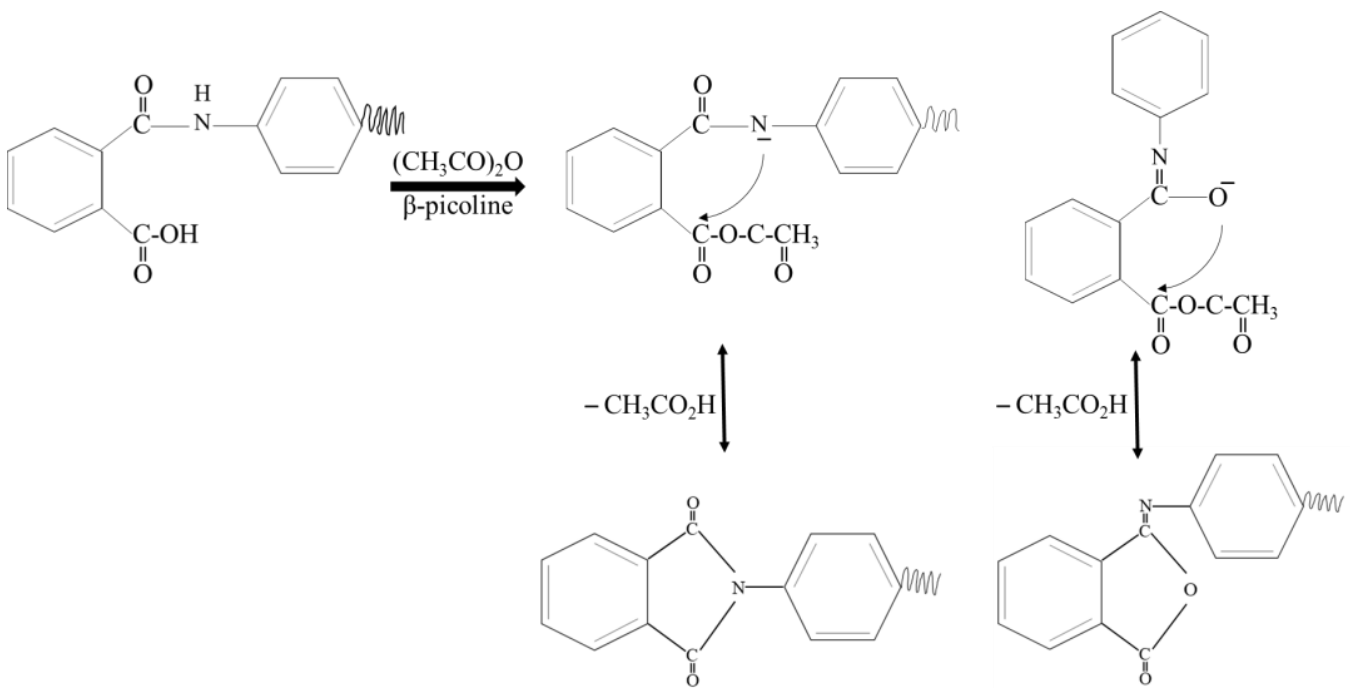

Isomer/Tautomer

Isomer/Tautomer II

Figure 3. Possible structure of polyimide formation via tautomerization[1].

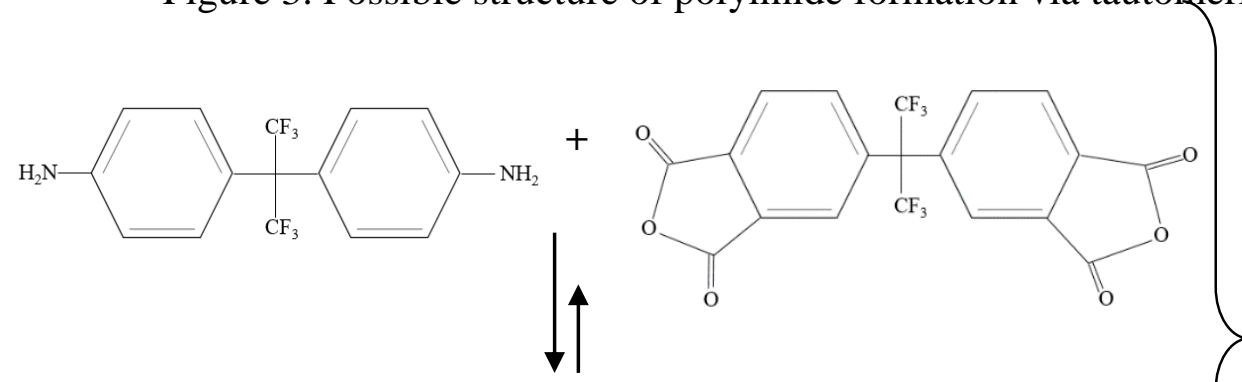

Step-1: Polyamic acids (PAAs)

formation

Equimolar monomer addition in amide solvent NMP under inert gas purging until

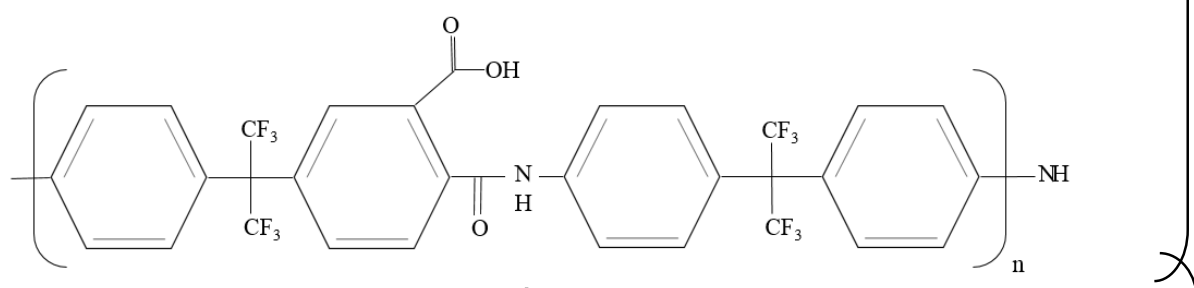
complete dissolution Solution of PAAs maintained at $20 \mathrm{wt} \%$ for $24 \mathrm{~h}$ to favor forward reaction.

Step-2:

Cyclodehydration via chemical imidization

Polyamic acid was

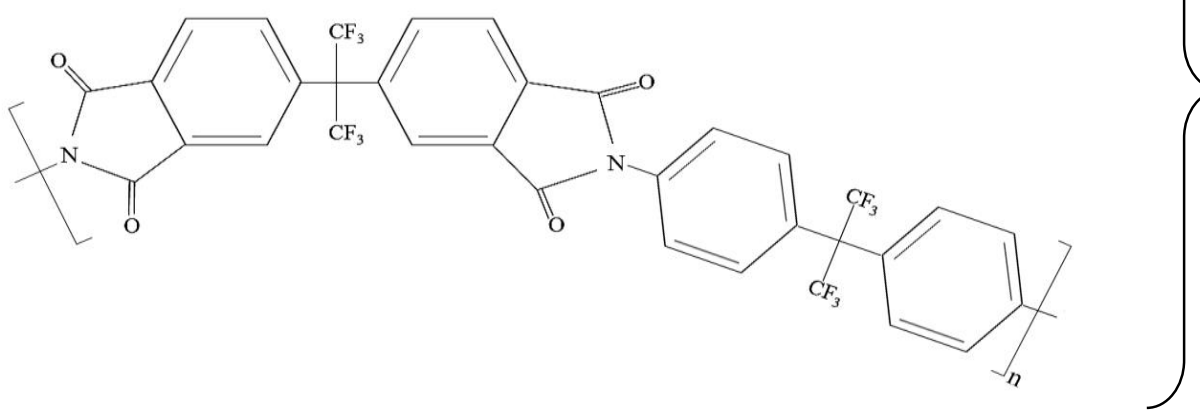
treated with imidizing mixture to form closed-ring PAAs, i.e. polyimide as the final product

Figure 4. 6FDA-6FpDA polyimide formation $[17,25]$. 


\section{Polymer Molecular Weight Study Using Gel Permeation Chromatography}

Five mg of sample was prepared by dissolving in THF and diluted to $5 \mu \mathrm{L} / \mathrm{mol}$ prior to sample injection to GPC (Agilent Technologies 1260 Infinity, PLgel 5 $\mu \mathrm{m}$ MIXED-C, Serial No. 0001022974-47) equipped with a light scattering detector. Polystyrene with Mw $100,000 \mathrm{~g} / \mathrm{mol}$ was used as the standard.

\section{Formation of Dense Polymeric Membrane for Gas Separation}

Solvent-induced casting was employed. The $20 \mathrm{wt} \%$ polymer dope was dissolved until homogeneous for $12 \mathrm{~h}$ and degassed for $2 \mathrm{hr}$. The glass plate was immersed in chloroform overnight prior to casting. The solvent evaporation was controlled in a glove box. Flat sheet membrane was fully formed after $48 \mathrm{~h}$.

\section{RESULTS AND DISCUSSION}

\section{Polymer Molecular Weight Revealed by Gel Permeation Chromatography}

The polymer molecular weight revealed by GPC is $4500 \mathrm{kDa}$ with polydispersity index of 1.48. This shows that a narrow distribution of PI was formed via chemical imidization. High molecular weight polyimide revealed by GPC showed that high degree imidization can be achieved via the chemical imidization route after post-synthesis treatment and physical purification.

\section{Imidization Degree of 6FDA-6FpDA Revealed by ${ }^{13} \mathrm{C}$ NMR and ${ }^{1} \mathrm{H}$ NMR}

${ }^{1} \mathrm{H}$ and ${ }^{13} \mathrm{C}$ NMR spectra were recorded with ECA JEOL Delta NMR (400 MHz), using tetramethylsilane (TMS) as an internal reference. $\mathrm{CDCl}_{3}$ was used as eluent to prepare the samples. Three samples consisted of monomers and a synthesised polymer were characterised and the results are shown below, which were in accordance with the previous literature [18-20]. Table 1 summarises the molecular arrangement of the desired functional moieties in the polyimide chain backbone. The formation of imide linkage $(\mathrm{C}$ $\mathrm{N})$ was detected at ${ }^{13} \mathrm{C}$ NMR, $\delta$ (ppm): $165.8(\mathrm{~s})$ and 131.9 (s). While for ${ }^{1} \mathrm{H}-\mathrm{NMR}$, the degree of imide conversion was calculated from the signal intensities of amic acid protons i.e., $-\mathrm{NH}$ and $-\mathrm{COOH} \delta(\mathrm{ppm})$ : 10.9-10.8. However, those peaks were undetected.

Table 1. Chemical shift for ${ }^{1} \mathrm{H}-\mathrm{NMR}$ and ${ }^{13} \mathrm{C}-\mathrm{NMR}$ for $6 \mathrm{FDA}-6 \mathrm{FpDA}$ polymer.

\begin{tabular}{|c|c|}
\hline Type of substance & Chemical shift $(\delta)$ \\
\hline \multirow[t]{3}{*}{$\begin{array}{l}\text { Polyimide: 6FDA- } \\
\text { 6FpDA polymer }\end{array}$} & $\begin{array}{l}{ }^{1} \mathrm{H} \text { NMR }\left(400 \mathrm{MHz}, \mathrm{CDCl}_{3}\right), \delta(\mathrm{ppm}): 8.21,8.05,7.91(\mathrm{t}), 3.715 \text {, } \\
2.5,1.8(\mathrm{~s})\end{array}$ \\
\hline & $\begin{array}{l}{ }^{13} \mathrm{C} \mathrm{NMR}\left(400 \mathrm{MHz}, \mathrm{CDCl}_{3}\right) \text { : } \\
\text { i. } 140 \text { ppm to } 117 \mathbf{p p m}(\text { upper band): } 138,136.67(\mathrm{~s}), 133.56 \text {, } \\
133.25(\mathrm{~d}), 132.8,131.9,130.3(\mathrm{~s}) 128,128.5(\mathrm{~d}) 127.2(\mathrm{~s}) \\
125.2,124.5,123.9,122.8(\mathrm{~m}), 120,19.45(\mathrm{~d})\end{array}$ \\
\hline & $\begin{array}{l}\text { ii. } 71 \text { ppm to } 61 \text { ppm (lower band): } 165.8(\mathrm{~s}), 67.4(\mathrm{~s}), 65.6 \text {, } \\
65.4,65.1,64.8,64.58,64.3,64.1,63.8(\mathrm{~m})\end{array}$ \\
\hline
\end{tabular}

\section{Determination of Imide Bands via ATR-FTIR}

The FTIR spectra of the conversion of polyamic acid to polyimide are shown in Figure 5 from PerkinElmer Frontier ${ }^{\mathrm{TM}}$ in a spectrum range of $4000-450 \mathrm{~cm}^{-1}$. 


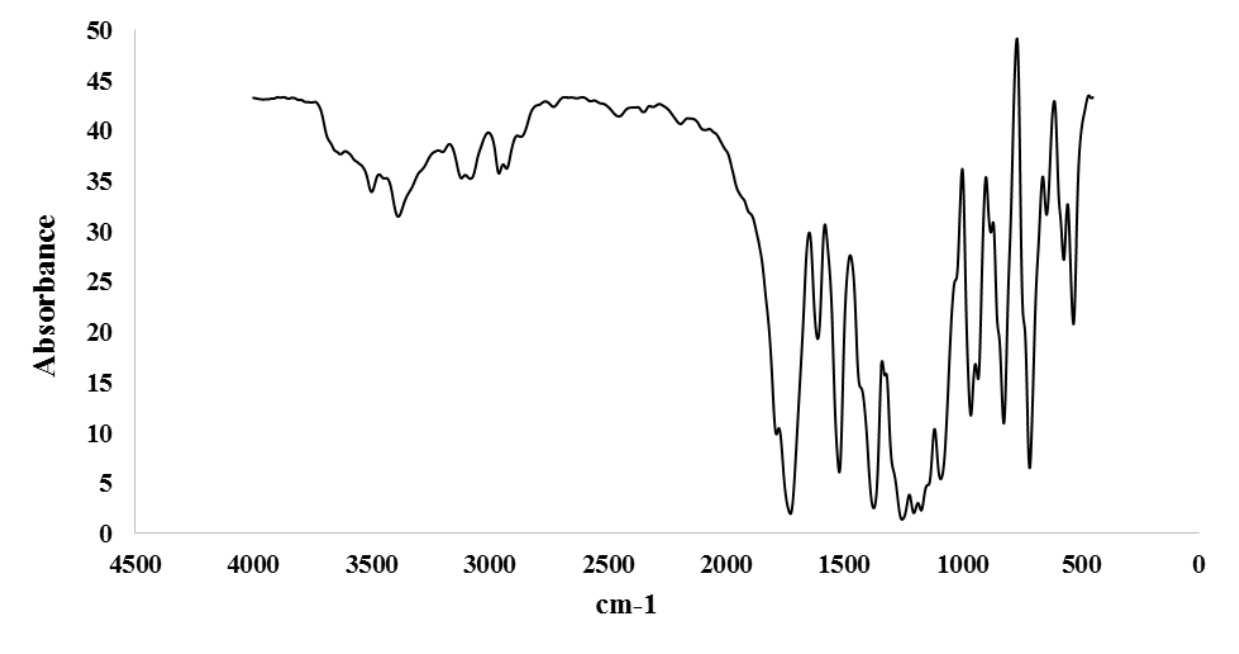

Figure 5. FTIR spectra of polyimide (6FDA-6FpDA).

The semi quantitative analysis by ATR-FTIR for as-synthesised 6FDA-6FpDA was performed to detect the degree of polyamic acid conversion to polyimide. The IR spectra revealed a small shoulder at $1788.37 \mathrm{~cm}-1$, denoting the formation of imide linkage $(\mathrm{C}-\mathrm{N}-\mathrm{C})$. The presence of amines absorption at $1728.58 \mathrm{~cm}-1$ (symmetric, $\mathrm{C}=\mathrm{O}$ ) and $1613.28 \mathrm{~cm}-1(\mathrm{C}=\mathrm{O})$ likely shows an indication of amic acids [26, 27]. These bands are generally observed in most PIs synthesised via chemical imidization even at a low intensity. After physical purification, the final product studied by NMR showed a certain degree of imidization. Through 13C NMR analysis, multiple splitting of carbon peak observed two sets of quartets at $\delta$ (ppm) $125.75,125.2,124.5$, and $123.9(\mathrm{~m})$ portraying the attached fluorine groups in both 6FDA and 6FpDA fragments [17, 28]. Imide linkage formation was detected at 165.8 (s) and 131.9 (s). The Mw of 6FDA-6FpDA synthesised via chemical imidization was determined via GPC analysis after purification of polymer was conducted. It was revealed that polyimide with a molecular weight $(\mathrm{Mw})$ of 4500 $\mathrm{kDa}$ and the polydispersity index of 1.48 was achieved.

\section{CONCLUSIONS}

Fluorinated polyimide (FPIs) i.e., 6FDA-6FpDA was successfully synthesised via a twostep polycondensation reaction employing catalytic cyclodehydration such as chemical imidization. Based on the degree of imidization studies via ATR-FTIR, ${ }^{1} \mathrm{H}$ and ${ }^{13} \mathrm{C}$ NMR showed a formation of imide linkages after thermal treatment during post-synthesis, while GPC analysis confirmed that FPIs having the molecular weight and dispersity index of $4500 \mathrm{kDa}$ and 1.48 , respectively, were obtained. Hence, the results from IR spectra, proton and carbon shift of NMR along with GPC showed that sufficient conversion degree of polyamic acid to polyimide had been achieved. Based on the polyimide precipitates recovered after microfiltration and recrystallisation, about $85 \%$ yield of FPIs was successfully produced. Furthermore, the intrinsic solubility of FPI produced was extraordinary, showing the existence of $-\mathrm{CF}_{3}$ in the polymer repeating unit. Due to this property, fabrication of flat sheet membrane can be easily made in a wide range of solvents. Based on the polymer's ease of processability and material properties, this polymer has a great prospect for gas separation application, especially to produce small 
footprint technology with superior separation performance of membrane from a lab to industrial scale.

\section{ACKNOWLEDGEMENTS}

This research work was supported by Universiti Teknologi PETRONAS under YUTP-FRG grant no. 0153AA-E08.

\section{REFERENCES}

[1] Ding M. Isomeric polyimides. Progress in Polymer Science. 2007;32:623-68.

[2] Liaw D-J, Wang K-L, Huang Y-C, Lee K-R, Lai J-Y, Ha C-S. Advanced polyimide materials: syntheses, physical properties and applications. Progress in Polymer Science. 2012;37:907-74.

[3] Misra AC, Tesoro G, Hougham G, Pendharkar SM. Synthesis and properties of some new fluorine-containing polyimides. Polymer. 1992;33:1078-82.

[4] Dine-Hart R, Wright W. Effect of structural variations on the thermo-oxidative stability of aromatic polyimides. Macromolecular Chemistry and Physics. 1972;153:237-54.

[5] Wang L, Zhao Z, Li J, Chen C. Synthesis and characterization of fluorinated polyimides for pervaporation of n-heptane/thiophene mixtures. European polymer journal. 2006;42:1266-72.

[6] Damaceanu M-D, Constantin C-P, Nicolescu A, Bruma M, Belomoina N, Begunov RS. Highly transparent and hydrophobic fluorinated polyimide films with ortho-kink structure. European polymer journal. 2014;50:200-13.

[7] Hougham G, Tesoro G, Viehbeck A. Influence of free volume change on the relative permittivity and refractive index in fluoropolyimides. Macromolecules. 1996;29:3453-6.

[8] Shioda T, Takamatsu N, Suzuki K, Shichijyo S. Influence of water sorption on refractive index of fluorinated polyimide. Polymer. 2003;44:137-42.

[9] Teerling OJ, McIntosh AC, Brindley J, Tam VHY. Premixed flame response to oscillatory pressure waves. Proceedings of the Combustion Institute. 2005;30:1733-40.

[10] Tanaka K, Kita H, Okano M, Okamoto K-i. Permeability and permselectivity of gases in fluorinated and non-fluorinated polyimides. Polymer. 1992;33:585-92.

[11] Tanaka K, Okano M, Kita H, Okamoto K-i, Nishi S. Effects of trifluoromethyl side groups on gas permeability and permselectivity in polyimides. Polymer journal. 1994;26:1186.

[12] Wang R, Cao C, Chung T-S. A critical review on diffusivity and the characterization of diffusivity of 6FDA-6FpDA polyimide membranes for gas separation. Journal of Membrane Science. 2002;198:259-71.

[13] Neyertz S, Brown D, Pandiyan S, van der Vegt NF. Carbon dioxide diffusion and plasticization in fluorinated polyimides. Macromolecules. 2010;43:7813-27.

[14] Pandiyan S, Brown D, Neyertz S, van der Vegt NF. Carbon dioxide solubility in three fluorinated polyimides studied by molecular dynamics simulations. Macromolecules. 2010;43:2605-21.

[15] Loh X, Daud M, Selamat M. Mechanical properties of kenaf/polypropylene composite: An investigation. Journal of Mechanical Engineering and Sciences. 2016;10:2098-110. 
[16] Tezara C, Siregar JP, Lim H, Fauzi F, Yazdi MH, Moey L, et al. Factors that affect the mechanical properties of kenaf fiber reinforced polymer: A review. Journal of Mechanical Engineering and Sciences. 2016;10:2159-75.

[17] Cornelius CJ, Marand E. Hybrid inorganic-organic materials based on a 6FDA6FpDA-DABA polyimide and silica: physical characterization studies. Polymer. 2002;43:2385-400.

[18] Hibshman C, Cornelius C, Marand E. The gas separation effects of annealing polyimide-organosilicate hybrid membranes. Journal of Membrane Science. 2003;211:25-40.

[19] Recio R, Palacio L, Prádanos P, Hernández A, Lozano AE, Marcos A, et al. Gas separation of 6FDA-6FpDA membranes: Effect of the solvent on polymer surfaces and permselectivity. Journal of Membrane Science. 2007;293:22-8.

[20] Tena A, Fernández L, Sánchez M, Palacio L, Lozano A, Hernández A, et al. Mixed matrix membranes of 6FDA-6FpDA with surface functionalized $\gamma$-alumina particles. An analysis of the improvement of permselectivity for several gas pairs. Chemical Engineering Science. 2010;65:2227-35.

[21] Kim J, Koros WJ, Paul DR. Physical aging of thin 6FDA-based polyimide membranes containing carboxyl acid groups. Part I. Transport properties. Polymer. 2006;47:3094-103.

[22] Kim T, Koros W, Husk G, O'brien K. "Reverse permselectivity" of N2 over CH4 in aromatic polyimides. Journal of applied polymer science. 1987;34:1767-71.

[23] Coleman M, Koros W. Isomeric polyimides based on fluorinated dianhydrides and diamines for gas separation applications. Journal of Membrane Science. 1990;50:285-97.

[24] Kailani M, Sung C, Huang S. Synthesis-Characterization of Model Imide Compounds and Chemical Imidization Studies. Polym Prepr. 1990;31:449-50.

[25] de Abajo J, Calle M, de la Campa JG, Garcia-Sanchez C, Lozano AE, Hernandez A, et al. 6FDA-6FpDA as a multipurpose polymer: Synthesis and influence of casting process on gas separation properties of ultrahigh MW 6FDA-6FpDA. American Chemical Society: USA; 2009.

[26] Al-Masri M, Kricheldorf HR, Fritsch D. New polyimides for gas separation. 1. Polyimides derived from substituted terphenylenes and 4,4'(hexafluoroisopropylidene) diphthalic anhydride. Macromolecules. 1999: 32(23):7853-8.

[27] Kailani MH, Sung CSP. Chemical imidization study by spectroscopic techniques. 2. polyamic acids. Macromolecules. 1998;31(17):5779-84.

[28] Cornelius CJ, Marand E. Hybrid inorganic-organic materials based on a 6FDA6FpDA-DABA polyimide and silica: physical characterization studies. Polymer (Guildf). 2002; 43(8):2385-400.

[29] Das M, Koros WJ. Performance of 6FDA-6FpDA polyimide for propylene/propane separations. Journal of Membrane Science. 2010; 365(12):399-408. 